\title{
Review
}

Pathophysiology

Diabetes Metab J 2014;38:100-106

http://dx.doi.org/10.4093/dmj.2014.38.2.100

pISSN 2233-6079 • eISSN 2233-6087

DIAB ET\&S \& METABOLISM JOUR N L

\section{The Role of Heat Shock Response in Insulin Resistance and Diabetes}

\author{
Tatsuya Kondo ${ }^{1}$, Hiroyuki Motoshima ${ }^{1}$, Motoyuki Igata ${ }^{1}$, Junji Kawashima ${ }^{1}$, Takeshi Matsumura ${ }^{1}$, Hirofumi Kai ${ }^{2}$, Eiichi Araki ${ }^{1}$ \\ Departments of ${ }^{1}$ Metabolic Medicine, ${ }^{2}$ Molecular Medicine, Kumamoto University Faculty of Life Sciences, Kumamoto, Japan
}

The expansion of life-style related diseases, such as metabolic syndrome (MS) and type 2 diabetes mellitus (T2DM), appears to be unstoppable. It is also difficult to cease their complications in spite of many antidiabetic medications or intervention of public administration. We and our collaborators found that physical medicine using simultaneous stimulation of heat with mild electric current activates heat shock response, thereby reducing visceral adiposity, insulin resistance, chronic inflammation and improving glucose homeostasis in mice models of T2DM, as well as in humans with MS or T2DM. This combination therapy exerts novel action on insulin signaling, $\beta$-cell protection and body compositions, and may provide a new therapeutic alternative in diabetic treatment strategy.

Keywords: Chronic inflammation; Cytoprotection; Diabetes mellitus; Heat-shock response

\section{INTRODUCTION}

Chronic metabolic diseases, such as obesity, type 2 diabetes mellitus (T2DM), metabolic syndrome (MS), dyslipidemia, and so on, are major causative factors for cardiovascular diseases. Among them, T2DM is a group of metabolic diseases characterized by hyperglycemia resulting from insulin resistance and insulin insufficiency. The affected population of T2DM is still increasing and the current treatment strategies may not enough to prevent vascular complications satisfactory. Insulin resistance is a major pathophysiological feature of T2DM, MS and obesity, and also associated with chronic inflammation, oxidative stress, endoplasmic reticulum (ER) stress, and cytokine production [1].

Heat shock (HS) is one of the major organismal and cellular stressors. Life form responds to HS in order to adapt environmental alterations. In 1962, Ritossa [2] identified the formation of a new chromosomal puffing pattern, which means transcriptional activation, against HS. This modification induces heat shock proteins (HSPs) synthesis in order to encounter cellular stress stimuli, such as HS, hypothermia, hypoxia, ischemia, heavy metal, ethanol, ultraviolet radiation, viral infection, and so on. The expression of HSP72 in T2MD is attenuated, and activation of heat shock response (HSR) confers benefits to improve lipid accumulation in liver and adipose tissue, inflammatory signals and insulin resistance, leading to metabolic ameliorations in diabetic rodents [3-8], monkey [9], and possibly in humans $[10,11]$.

In this review, we focus on the relationship between HSR activation and chronic inflammation, insulin resistance, metabolic profiles in MS and T2DM. More importantly, we explore the benefits of novel treatment modality with a combination of HS and mild electrical stimulation (MES) that activate HSR efficiently and may target other molecules, providing new insights into physical medicine.
Corresponding author: Tatsuya Kondo

Department of Metabolic Medicine, Kumamoto University Faculty of Life

Sciences, Honjo 1-1-1, Chuo Ward, Kumamoto 860-8556, Japan

E-mail: t-kondo@gpo.kumamoto-u.ac.jp
This is an Open Access article distributed under the terms of the Creative Commons Attribution Non-Commercial License (http://creativecommons.org/licenses/by-nc/3.0/) which permits unrestricted non-commercial use, distribution, and reproduction in any medium, provided the original work is properly cited. 


\section{THE EFFECTS OF HSR ACTIVATION ON DIABETIC MODEL MICE}

\section{Mild electrical stimulation with hyperthermia}

We found that activation of HSR using mild electric current with specific pulse wave and frequency in heat stimulation of 42 degrees, maximally induced HSP72, which is a major HSR product to exert HSR effects. HSP72 acts as a molecular chaperone to restrain the formation of abnormal protein aggregation, and controls protein transportation, cell death, chronic inflammation to protect cells from various kinds of stresses [12].

It has been reported that the expression of HSP72 was decreased in T2DM patients having insulin resistance, and that insulin resistance and glucose metabolism were both improved when HSP72 restored by various kinds of methods, such as HSP72 transgenic [4], HSP72 inducer administration [5,9,13] or long-term hyperthermia [6,7,11]. Our method (mild electrical stimulation with hyperthermia, MET) reduced fasting plasma glucose (Fig. 1A), insulin levels, insulin resistance (Fig. 1C), the amount of visceral adiposity, hepatic steatosis (Fig. 1D), and chronic inflammatory cytokines, with amelioration of insulin signaling (Fig. 1F, G) and whole body glucose homeostasis (Fig. 1B, E) in mice models of T2DM $[8,14]$.

This MET also protects pancreatic $\beta$-cells from high glucose and cellular stresses, such as ER stress or oxidative stress [14]. When MIN6 cells are treated with tumor necrosis factor $\alpha$ (TNF- $\alpha$ ), one of the stress kinase, $c$-jun $\mathrm{N}$-terminal kinase (JNK) was activated (phosphorylated). This was largely eliminated using MET accompanied by attenuation of ER stress markers and cell death signals in TNF- $\alpha$ stimulated MIN6 cells (Fig. 2A). In insulin resistance with insulin deficient $d b / d b$ mice, MET also exerts antidiabetic (Fig. 2B, C) and antiapoptotic effects in pancreatic $\beta$-cells with HSP72 induction, insulin and PDX-1 enhancement (Fig. 2D-H) [14]. The reinforcement of HSP72 in pancreatic $\beta$-cells may protect cells from apoptotic events and maintain the $\beta$-cell mass in diabetic patients.
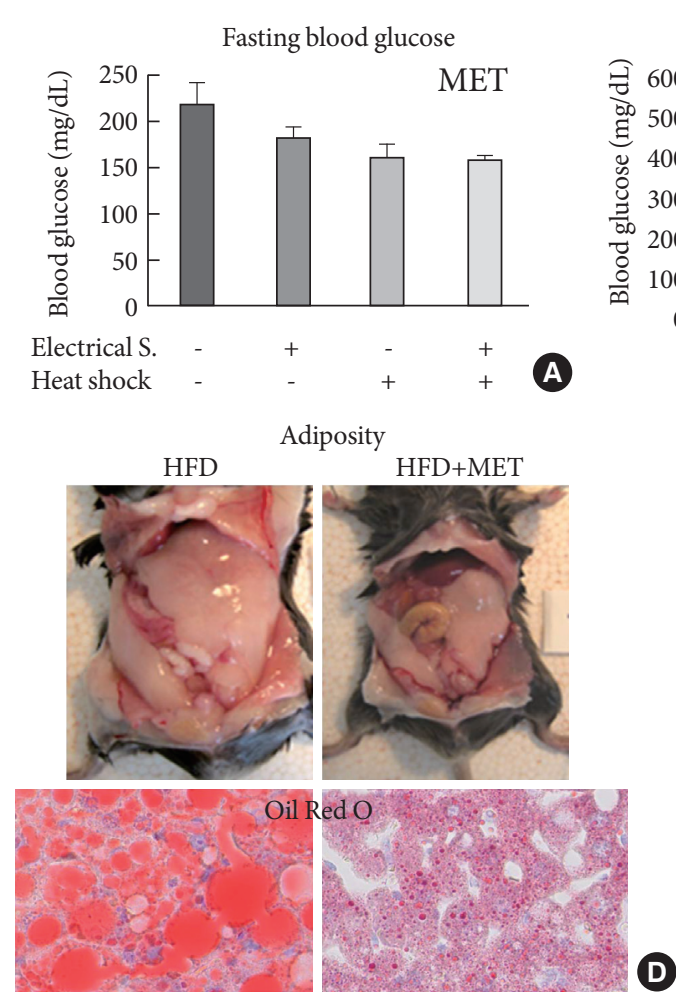
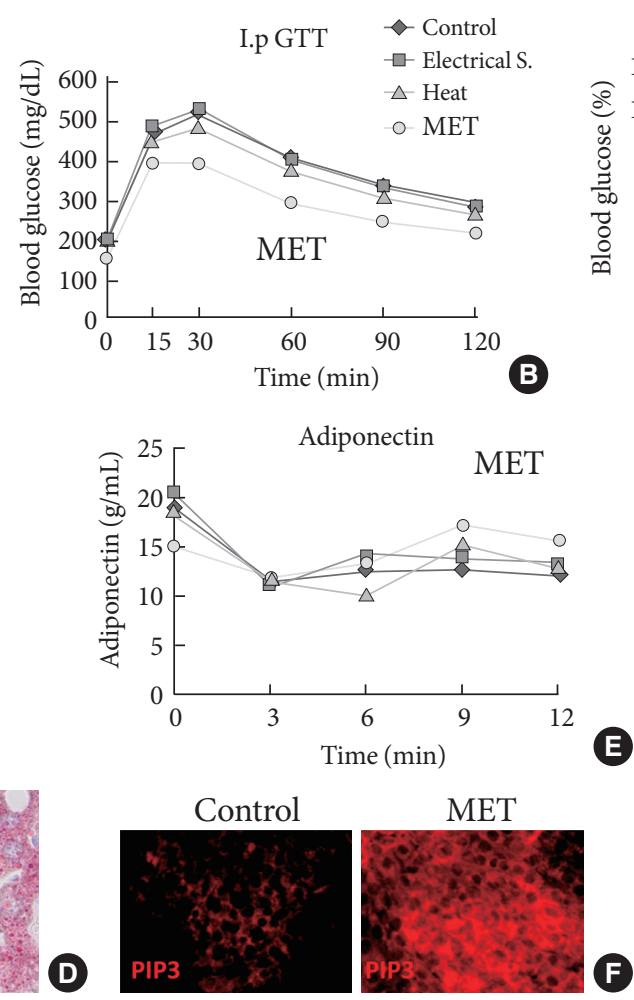
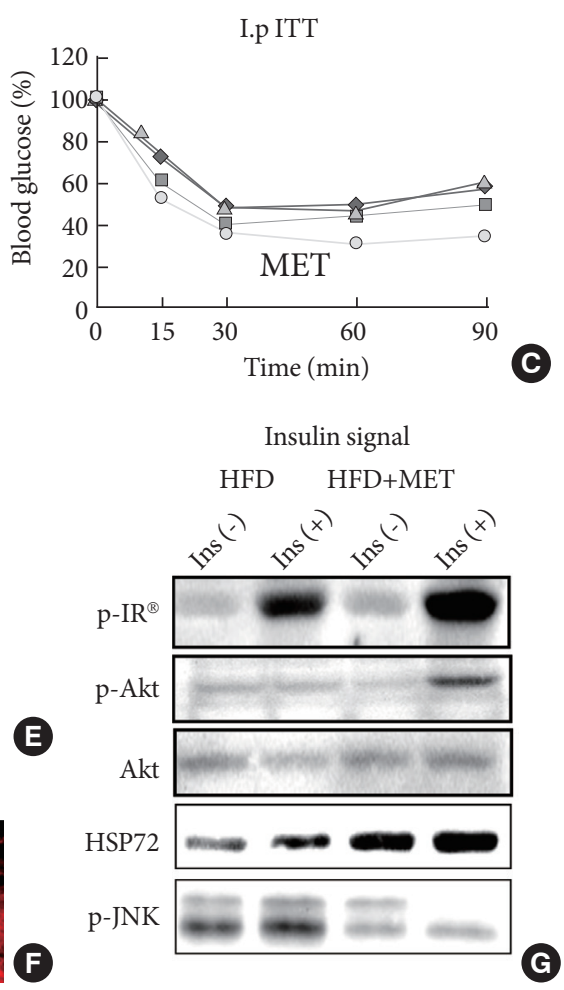

Fig. 1. Heat shock response activation by mild electrical stimulation with hyperthermia (MET) improves insulin resistance. MET using simultaneous heat shock with mild electrical stimulation decreases fasting plasma glucose (A) and improves glucose tolerance (B) and insulin resistance (C) accompanied by reduction of visceral adiposity and hepatic steatosis (D). Circulating adiponectin is increased (E) and hepatic insulin signal is restored with HSP72 induction and c-jun N-terminal kinase (JNK) attenuation by MET (F, G). I.p, intraperitoneal; GTT, glucose tolerance test; ITT, insulin tolerance test; HFD, high fat diet. Adapted from Morino S, et al. Mild electrical stimulation with heat shock ameliorates insulin resistance via enhanced insulin signaling. PLoS One 2008;3:e4068 [8]. 

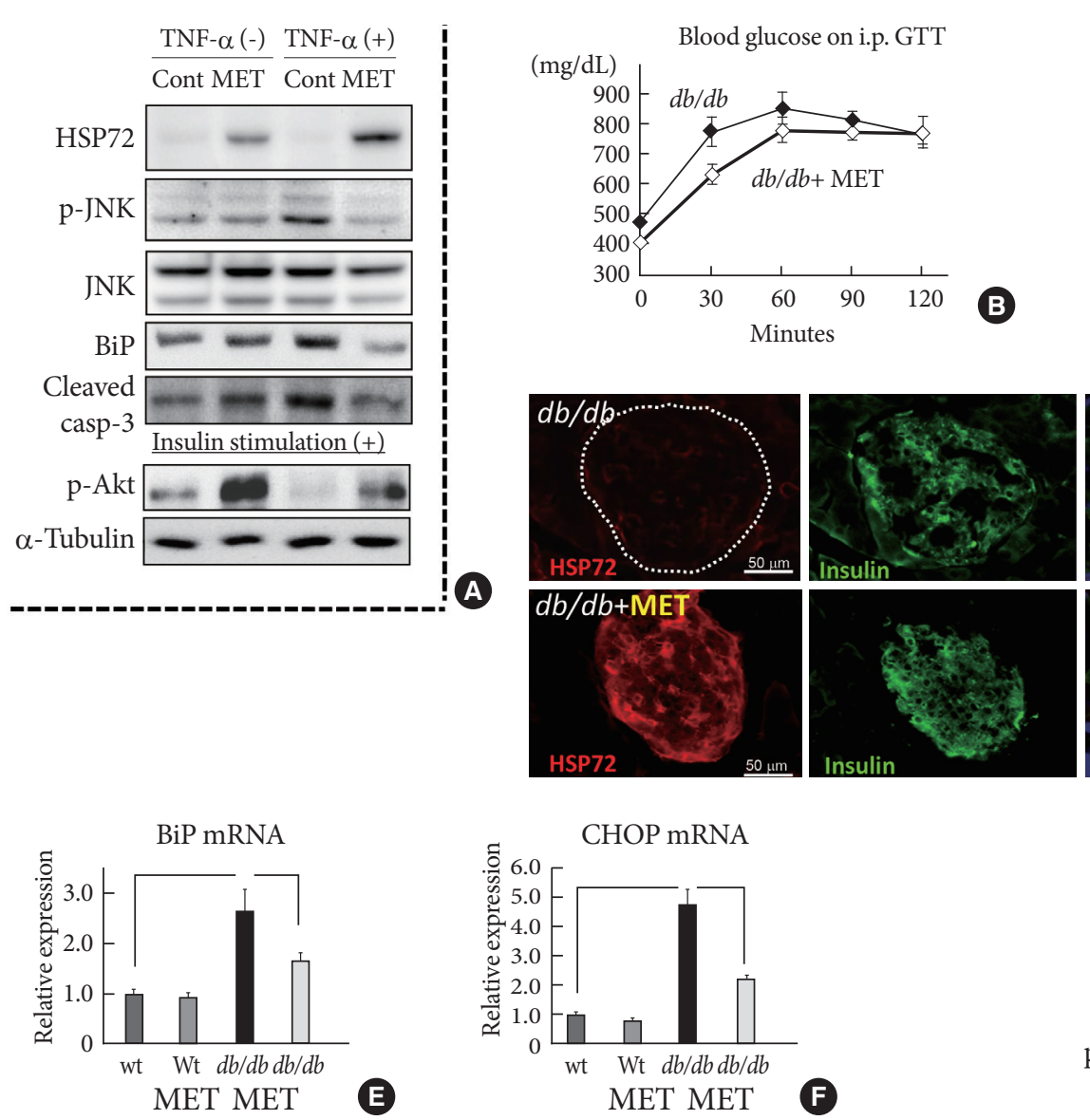

Fig. 2. Heat shock response activation by mild electrical stimulation with hyperthermia (MET) protects pancreatic $\beta$-cells. Although c-jun $\mathrm{N}$-terminal kinase (JNK), endoplasmic reticulum stress marker BiP and death signal caspase- 3 are activated in tumor necrosis factor $\alpha$ (TNF- $\alpha$ ) stimulated MIN6 cells, MET treatment attenuates these abnormal activations (A). Akt phosphorylation is partially restored by MET as well (A). MET treatment alleviates glucose dysregulation (B) with insulin insufficiency (C) in aged $d b / d b$ mice. This therapy protects pancreatic $\beta$-cells from apoptotic signals and increases PDX-1 nuclear expression (D-H). I.p, intraperitoneal; GTT, glucose tolerance test. Adapted from Kondo T, et al. Diabetes 2012;61:838-47 [14].
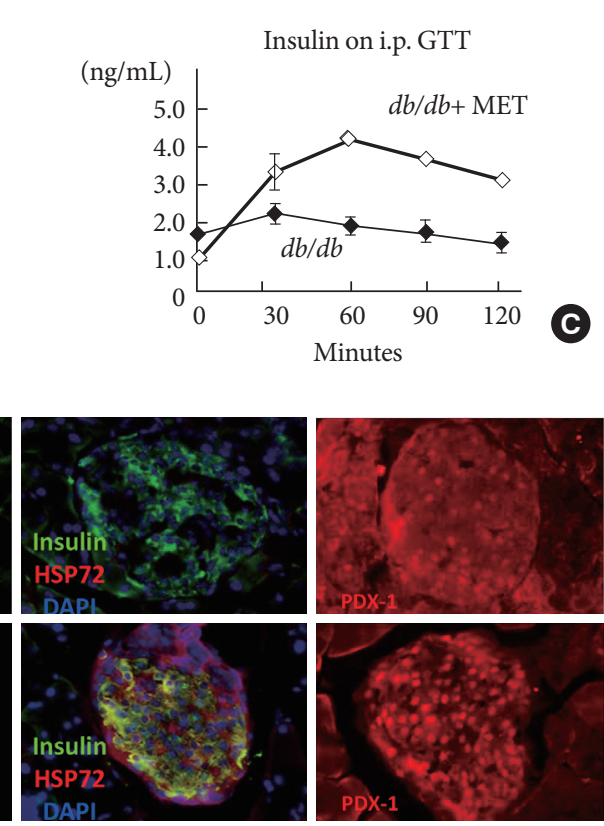

(D)

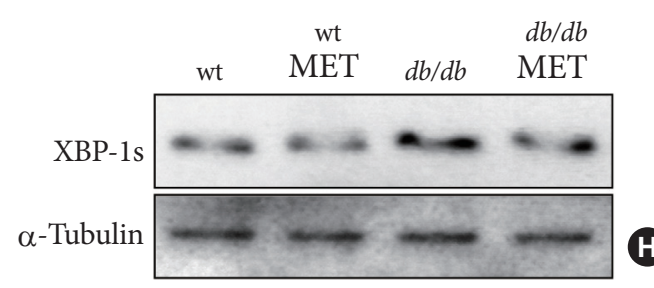

\section{HSP72 inducer geranylgeranyl acetone}

Besides MET, a HSP72 inducible medication, geranylgeranyl acetone (GGA) can be used to activate HSR. Antigastric ulcer drug, GGA (Fig. 3A) was developed in Japan (generic name: teprenone) and is widely used in clinic. It is identified that gastric mucosa protection effect by GGA depends on HSP72 expression.

When a high-fat fed diabetes model mouse was given GGA orally every day in $200 \mathrm{mg} / \mathrm{kg} / \mathrm{day}$, the metabolic and pathophysiological effects appeared to be quite similar to MET treatment including weight reduction, reduced visceral fat accumu- lation, improvement of insulin resistance and glucose tolerance (Fig. 3B, C), and reduction in chronic inflammation [3].

GGA administration induced HSP72 mainly in liver (Fig. 3D), reduced visceral adipocyte size (Fig. 3H), improved insulin resistance and JNK activation (Fig. 3E). Hepatic gluconeogenic enzymes, such as phosphoenolpyruvate carboxykinase or glucose 6-phosphatase mRNA levels were both decreased by GGA treatment (Fig. 3F, G) [3]. These observations indirectly prove that MET improves body composition and glucose metabolism abnormality mainly through HSP72 induction. 

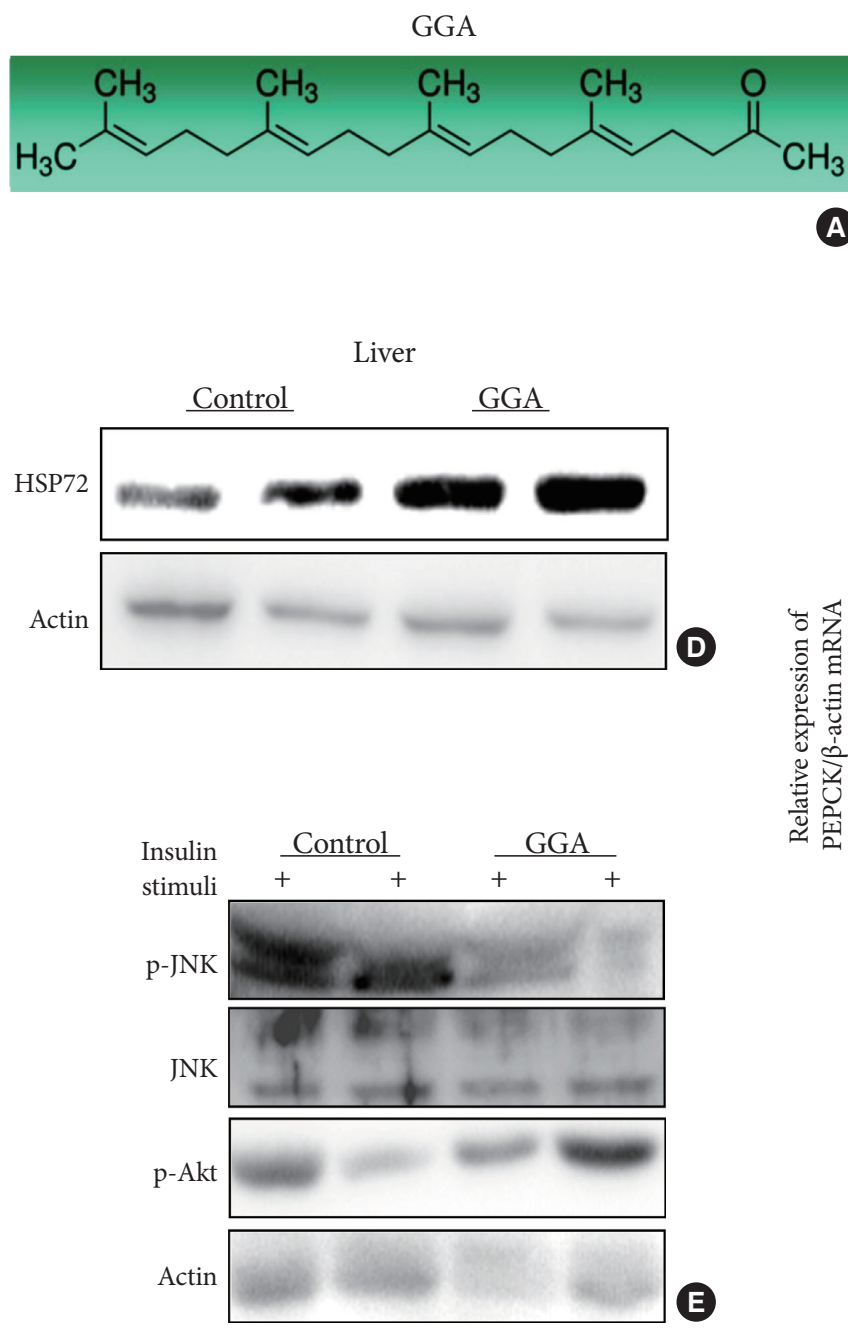
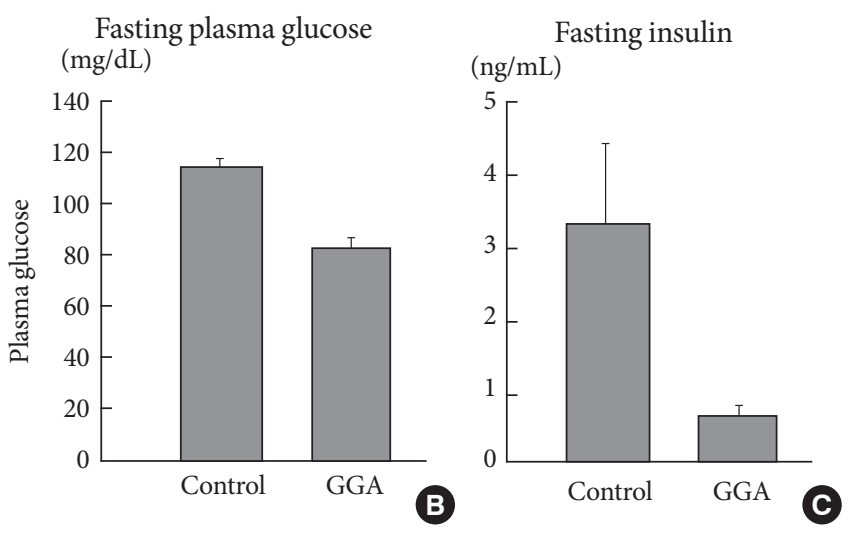

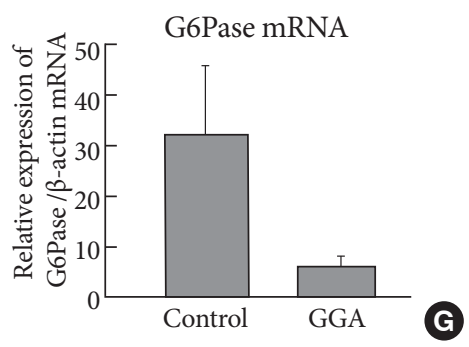

Epididymal fat

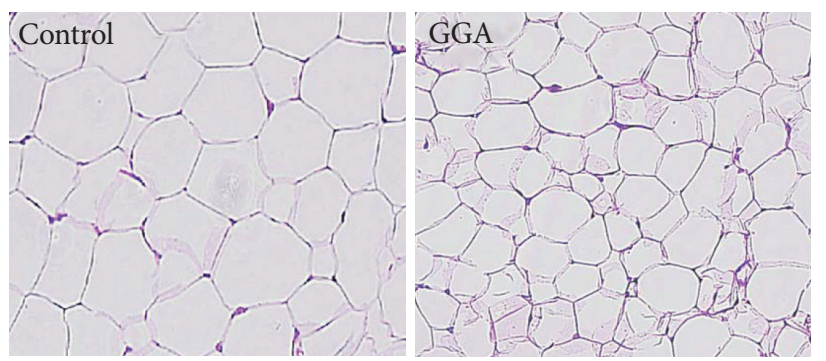

$\boldsymbol{\Theta}$

Fig. 3. Heat shock response activation by geranylgeranyl acetone (GGA) (A) improves insulin resistance. HSP72 expression is mainly increased in liver (D), and fasting plasma glucose (B), insulin levels (C) and insulin resistance are ameliorated with reductions in hepatic gluconeogenic enzymes (F, G) by the GGA oral administration. GGA treatment decreases visceral adipocyte size $(\mathrm{H})$, c-jun $\mathrm{N}$-terminal kinase (JNK) activation and restores insulin signaling (E). PEPCK, phosphoenolpyruvate carboxykinase; G6P, glucose 6-phosphatase. Adachi H, et al. Am J Physiol Endocrinol Metab 2010;299:E764-71, with permission from the American Physiological Society [3].

\section{THE EFFECTS OF HSR ACTIVATION ON HUMANS}

\section{Normal subjects}

We performed the safe confirmation trial employing normal subjects before applying MET to life style related diseases in humans with MS or T2DM (Fig. 4). MET device specifically designed for humans produces electrical stimulation of $1.4 \pm 0.1 \mathrm{~V} / \mathrm{cm}$ : the pads were positioned on the front and back of the abdomen, 55 pulses per second, 0.1 millisecond duration with $42^{\circ} \mathrm{C}$ heat. The padded area was $15 \mathrm{~cm}$ in length $\times 25 \mathrm{~cm}$ in width.
The harm phenomenon was not recognized without affecting the body composition, glucose metabolism and insulin resistance as expected, but the inflammatory markers in the normal range level showed a significant reduction, unexpectedly [10]. This physiologic significance was unidentified, but it is possible that MET is an effective anti-inflammatory intervention to alleviate low-level chronic inflammation and may contribute to decrease arteriosclerosis risk in the long term.

\section{MS subjects}

Clinical trial to investigate the effects of MET on MS was per- 


\begin{tabular}{|c|c|c|c|c|c|}
\hline Modality & MET & GGA & MET & MET & MET \\
\hline Target & \multicolumn{2}{|c|}{ Diabetic model mice } & Healthymales & Metabolic syndrome & Type 2 diabetes \\
\hline - Body weight & $\rightarrow$ & $\downarrow$ & $\rightarrow$ & $\rightarrow$ & $\rightarrow$ \\
\hline - Abdominal fat & $\downarrow$ & $\downarrow$ & $\rightarrow$ & $\downarrow$ & $\downarrow$ \\
\hline - Insulin resistance & $\downarrow$ & $\downarrow$ & $\rightarrow$ & $\downarrow$ & $\downarrow$ \\
\hline - JNK & $\downarrow$ & $\downarrow$ & ND & ND & ND \\
\hline - Inflammatory cytokines & $\downarrow$ & $\downarrow$ & $\downarrow$ & $\downarrow$ & $\downarrow$ \\
\hline - $\beta$-Cell failure & $\downarrow$ & ND & $\rightarrow$ & Protective (?) & Protective (?) \\
\hline
\end{tabular}

Fig. 4. The summarized effects of mild electrical stimulation with hyperthermia (MET) on metabolic diseases. MET treatment or geranylgeranyl acetone (GGA) administration decreases the amount of visceral fat, insulin resistance, c-jun N-terminal kinase (JNK) activation, inflammatory cytokines and contributes to protect pancreatic $\beta$-cells in mice models of diabetes. In normal human subjects, MET exerts anti-inflammatory effect in normal range. MET exerts antidiabetic, antiobesity, and anti-inflammatory effects in metabolic syndrome or type 2 diabetes mellitus subjects. These observations are quite similar to those in mice models. ND, not determined.

formed. Forty of eligible MS male subjects were randomly assigned into two groups; group I, a 12-week intervention period of MET followed by 12 weeks with no treatment; or group II, the reversed order. During the MET-treatment period, subjects were instructed to use MET 4 times a week for $60 \mathrm{~min}$ utes per session.

Visceral adipose volume measured by abdominal computed tomography scan and waist circumference was reduced, whereas body weight and subcutaneous adipose volume were not. Both systolic and diastolic blood pressures were significantly reduced. Fasting blood glucose and fasting insulin levels were both decreased. HbA1c showed a trend of reduction, but could not reach significance. As glucose and insulin levels were reduced, homeostatic model assessment of insulin resistance, quantitative insulin sensitivity check index, and composite whole body insulin sensitivity index were all significantly improved, and insulinogenic index was significantly decreased. Inflammatory cytokines or adipokines, such as high sensitivity C-reactive protein, adiponectin, leptin, and TNF- $\alpha$, were all improved. In addition with those beneficial results, the count of white blood cells and low densitiy lipoprotein cholesterol were decreased (Fig. 4).

The mechanisms in reduction of visceral adiposity may be due to activation of 5' AMP-activated protein kinase (AMPK), upregulation of peroxisome proliferator-activated receptor coactivator- $1 \alpha$ with increased mitochondrial density, JNK inhibition and/or muscle hypertrophy via nuclear factor of acti- vated T-cell signal activation.

Inactivation of JNK and inhibition of nuclear factor- $\kappa B$ $(\mathrm{NF}-\kappa \mathrm{B})$ nuclear translocation may contribute to attenuate chronic inflammation.

\section{T2DM patients}

Similar protocol was employed to investigate the MET effects in 40 of obese T2DM patients. Almost similar beneficial results were obtained with significant reduction of HbAlc in this cohort. Decrease of urinary albumin excretion and improvement of lipid profiles were observed (Fig. 4). As MET exerts antidiabetic, antiobesity effects as well as anti-inflammatory effect, this method may contribute to decrease the risk of atherogenic complications, such as brain infarction or ischemic heart disease.

\section{THE MECHANISMS OF MET EFFECTS ON METABLIC DISEASES}

Given that the combination of warm temperature and MES is administered to abdominal area, it is possible that abdominal organs, including liver, visceral fat, pancreatic islets and /or intestine are very likely to be targets. In mouse models, suppression of hepatic gluconeogenesis and reduction of visceral adiposity were both apparent.

A great number of humoral factors in blood circulation or neurotransmitters interact from one organ to another. Thus, 
interorgan crosstalk may regulate whole body glucose homeostasis. Then, we focused on circulating monocytes, which may reflect systemic inflammatory status.

To identify the effects of MET on the inflammatory milieu in vivo, CD14 positive circulating monocytes were isolated and levels of cytokine expression were examined. After 4 weeks of MET treatment, HSP72 protein and phosphorylation of AMPK in monocytes significantly increased. Phospho-JNK and lipopolysaccharide (LPS)-stimulated NF- $\kappa \mathrm{B}$ p65 nuclear translocation was attenuated. Quantitative measurements of mRNA expression after LPS stimulation for C-reactive protein, interleukin-6, NF- $\kappa \mathrm{B}$, and TNF- $\alpha$ showed a significant reduction. These results clearly indicate that one of the target tissues of MET treatment is monocytes; however, it is also possible that other solid organs such as liver, adipose tissues and pancreas, may be targets as well. We cannot exclude the possibility that the changes in monocytes character might be secondary following amelioration of glucose metabolism.

\section{CONCLUSIONS}

One of the distinctive features of our method is using MES, which is specifically designed to act on human body, but not causing muscle contraction. It has been reported that a certain mild electric current increases blood flow or enhances wound healing [15]. Our collaborators group identified a particular mild electric current (direct current, $1.4 \pm 0.1 \mathrm{~V} / \mathrm{cm}$ between the pad distance, 55 pulses per second, 0.1 millisecond duration) significantly activates insulin receptor accumulation in lipid raft component [16], there by increasing Akt phosphoryration and enhance glucose uptake independent of insulin [17]. They also found that this current suppresses HSP72 protein proteosomal degradation to increase the protein levels of HSP72 [18]. Therefore, this combination of $42^{\circ} \mathrm{C}$ heat and particular mild electrical current may maximize the HSR effects to improve metabolic abnormalities in life-style related diseases, such as MS or T2DM. As these mechanisms, including antidiabetic, antiobesity and anti-inflammatory signals [19], are completely different from present medical approaches, MET could be one of the novel therapeutic alternatives, which can be added on the current therapy to T2DM.

\section{CONFLICTS OF INTEREST}

No potential conflict of interest relevant to this article was re- ported.

\section{REFERENCES}

1. Ueki K, Kondo T, Kahn CR. Suppressor of cytokine signaling 1 (SOCS-1) and SOCS-3 cause insulin resistance through inhibition of tyrosine phosphorylation of insulin receptor substrate proteins by discrete mechanisms. Mol Cell Biol 2004;24:5434-46.

2. Ritossa F. A new puffing pattern induced by temperature shock and DNP in Drosophila. Experientia 1962;18:571-3.

3. Adachi H, Kondo T, Ogawa R, Sasaki K, Morino-Koga S, Sakakida M, Kawashima J, Motoshima H, Furukawa N, Tsuruzoe K, Miyamura N, Kai H, Araki E. An acylic polyisoprenoid derivative, geranylgeranylacetone protects against visceral adiposity and insulin resistance in high-fat-fed mice. Am J Physiol Endocrinol Metab 2010;299:E764-71.

4. Chung J, Nguyen AK, Henstridge DC, Holmes AG, Chan MH, Mesa JL, Lancaster GI, Southgate RJ, Bruce CR, Duffy SJ, Horvath I, Mestril R, Watt MJ, Hooper PL, Kingwell BA, Vigh L, Hevener A, Febbraio MA. HSP72 protects against obesity-induced insulin resistance. Proc Natl Acad Sci U S A 2008;105: 1739-44.

5. Gupte AA, Bomhoff GL, Morris JK, Gorres BK, Geiger PC. Lipoic acid increases heat shock protein expression and inhibits stress kinase activation to improve insulin signaling in skeletal muscle from high-fat-fed rats. J Appl Physiol (1985) 2009;106: 1425-34.

6. Gupte AA, Bomhoff GL, Swerdlow RH, Geiger PC. Heat treatment improves glucose tolerance and prevents skeletal muscle insulin resistance in rats fed a high-fat diet. Diabetes 2009;58: 567-78.

7. Kokura S, Adachi S, Manabe E, Mizushima K, Hattori T, Okuda T, Nakabe N, Handa O, Takagi T, Naito Y, Yoshida N, Yoshikawa T. Whole body hyperthermia improves obesity-induced insulin resistance in diabetic mice. Int J Hyperthermia 2007;23:259-65.

8. Morino S, Kondo T, Sasaki K, Adachi H, Suico MA, Sekimoto E, Matsuda T, Shuto T, Araki E, Kai H. Mild electrical stimulation with heat shock ameliorates insulin resistance via enhanced insulin signaling. PLoS One 2008;3:e4068.

9. Kavanagh K, Flynn DM, Jenkins KA, Zhang L, Wagner JD. Restoring HSP70 deficiencies improves glucose tolerance in diabetic monkeys. Am J Physiol Endocrinol Metab 2011;300:E894-901.

10. Kondo T, Sasaki K, Adachi H, Nakayama Y, Hatemura M, Matsuyama R, Tsuruzoe K, Furukawa N, Motoshima H, Mori- 
no Koga S, Yamashita Y, Miyamura N, Kai H, Araki E. Heat shock treatment with mild electrical stimulation safely reduced inflammatory markers in healthy male subjects. Obes Res Clin Pract 2010;4:e83-162.

11. Hooper PL. Hot-tub therapy for type 2 diabetes mellitus. N Engl J Med 1999;341:924-5.

12. Chow AM, Steel R, Anderson RL. Hsp72 chaperone function is dispensable for protection against stress-induced apoptosis. Cell Stress Chaperones 2009;14:253-63.

13. Literati-Nagy B, Kulcsar E, Literati-Nagy Z, Buday B, Peterfai E, Horvath T, Tory K, Kolonics A, Fleming A, Mandl J, Korányi $\mathrm{L}$. Improvement of insulin sensitivity by a novel drug, BGP-15, in insulin-resistant patients: a proof of concept randomized double-blind clinical trial. Horm Metab Res 2009;41: 374-80.

14. Kondo T, Sasaki K, Matsuyama R, Morino-Koga S, Adachi H, Suico MA, Kawashima J, Motoshima H, Furukawa N, Kai H, Araki E. Hyperthermia with mild electrical stimulation protects pancreatic beta-cells from cell stresses and apoptosis. Diabetes 2012;61:838-47.

15. Zhao M, Song B, Pu J, Wada T, Reid B, Tai G, Wang F, Guo A, Walczysko P, Gu Y, Sasaki T, Suzuki A, Forrester JV, Bourne
HR, Devreotes PN, McCaig CD, Penninger JM. Electrical signals control wound healing through phosphatidylinositol3-OH kinase-gamma and PTEN. Nature 2006;442:457-60.

16. Morino-Koga S, Yano S, Kondo T, Shimauchi Y, Matsuyama S, Okamoto Y, Suico MA, Koga T, Sato T, Shuto T, Arima H, Wada I, Araki E, Kai H. Insulin receptor activation through its accumulation in lipid rafts by mild electrical stress. J Cell Physiol 2013;228:439-46.

17. Yano S, Morino-Koga S, Kondo T, Suico MA, Koga T, Shimauchi Y, Matsuyama S, Shuto T, Sato T, Araki E, Kai H. Glucose uptake in rat skeletal muscle L6 cells is increased by low-intensity electrical current through the activation of the phosphatidylinositol-3-OH kinase (PI-3K) / Akt pathway. J Pharmacol Sci 2011;115:94-8.

18. Morino S, Suico MA, Kondo T, Sekimoto E, Yano S, Matsuda T, Matsuno T, Shuto T, Araki E, Kai H. Mild electrical stimulation increases ubiquitinated proteins and Hsp72 in A549 cells via attenuation of proteasomal degradation. J Pharmacol Sci 2008;108:222-6.

19. Hooper PL, Hooper PL. Inflammation, heat shock proteins, and type 2 diabetes. Cell Stress Chaperones 2009;14:113-5. 